\title{
Emergency radiology
}

\section{Medical emergencies}

\author{
Oscar Craig \\ F.R.C.S.I F.F.R.
}

Consultant Radiologist,

St Mary's Hospital, London, W.2, and Bolingbroke Hospital, London, S.W.11

THE RADIOLOGY of some common surgical emergencies seen by the Casualty Officer was described in Part I. This paper considers the radiology of some of the common medical conditions.

\section{Pneumothorax (Fig. 1)}

A pneumothorax may be either traumatic, spontaneous or artificial. The latter is rarely a problem to the Casualty Officer. Three varieties of pneumothorax are described:

(a) Open. In this the wound communicates with the pleural cavity.

(b) Closed. This is the most common type and the air in the pleural cavity is gradually absorbed.

(c) Valvular. In this variety as air drawn into the pleural cavity cannot escape on expiration, tension increases resulting in mediastinal displacement and acute respiratory distress.

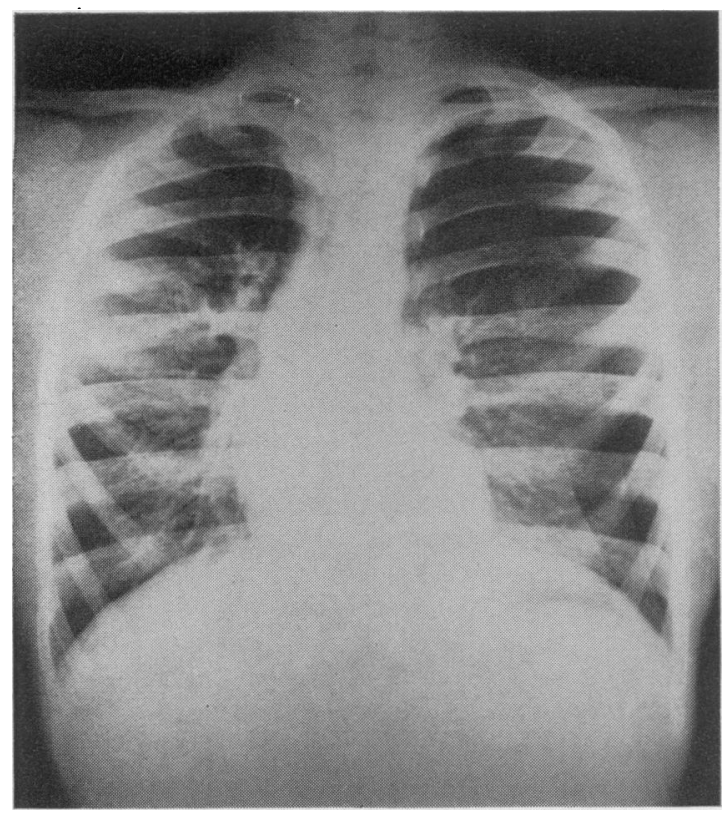

FIG. 1. Left-sided pneumothorax.

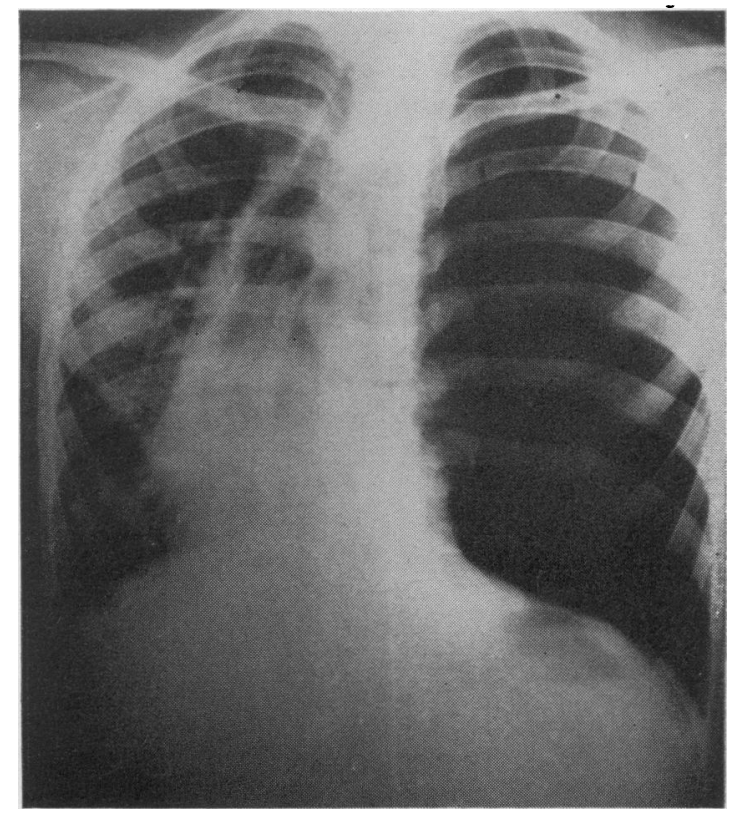

FIG. 2. Left-sided tension pneumothorax with medistinal shift to the right.

Radiological investigation consists of a posteroanterior film of the chest. If a pneumothorax is not seen, but is clinically strongly suspected, a further film in expiration should be taken. This will reveal many small pneumothoraces readily missed on a single inspiratory film.

On the affected side the lung edge can be seen inside the rib cage and parallel to it. Beyond the lung edge there are no vascular markings and there is an increased translucency. Depending on the quantity of air in the pneumothorax varying degrees of lung compression are seen. Tension pneumothorax produces displacement of the mediastinum to the opposite side (Fig. 2). Screening or films in inspiration and expiration may demonstrate a shift of the mediastinum to the affected side on inspiration and back to the unaffected side on expiration. 
In the vast majority of cases of spontaneous pneumothorax, no underlying chest pathology can be identified, and these cases are thought to be due to the rupture of a small emphysematous bulla. Generalized emphysema rarely gives rise to a spontaneous pneumothorax, but localized emphysema, secondary to tuberculosis, pneumoconiosis, sarcoidosis, etc., may do so. Spontaneous pneumothorax has also been described complicating both primary and secondary bronchial neoplasms. Patients with severe asthma may develop a spontaneous pneumothorax during an attack.

Traumatic pneumothorax is frequently accompanied by traumatic lesions to the bones. Tears of the diaphragm, ruptures of the oesophagus and 'fractures' of the trachea or bronchi may produce a pneumothorax. Mediastinal emphysema may be present also, in these conditions.

A pneumothorax must be distinguished from bullous emphysema. The presence of septa passing between the cysts and extending out to the chest wall is seen in bullous emphysema (Fig. 3).

A hydropneumothorax or a haemopneumothorax is recognized by the horizontal fluid level seen in the hemithorax passing from the lateral chest wall to the lung edge. It is not infrequently seen following chest surgery or as a complication of chest trauma. It can also result from infection

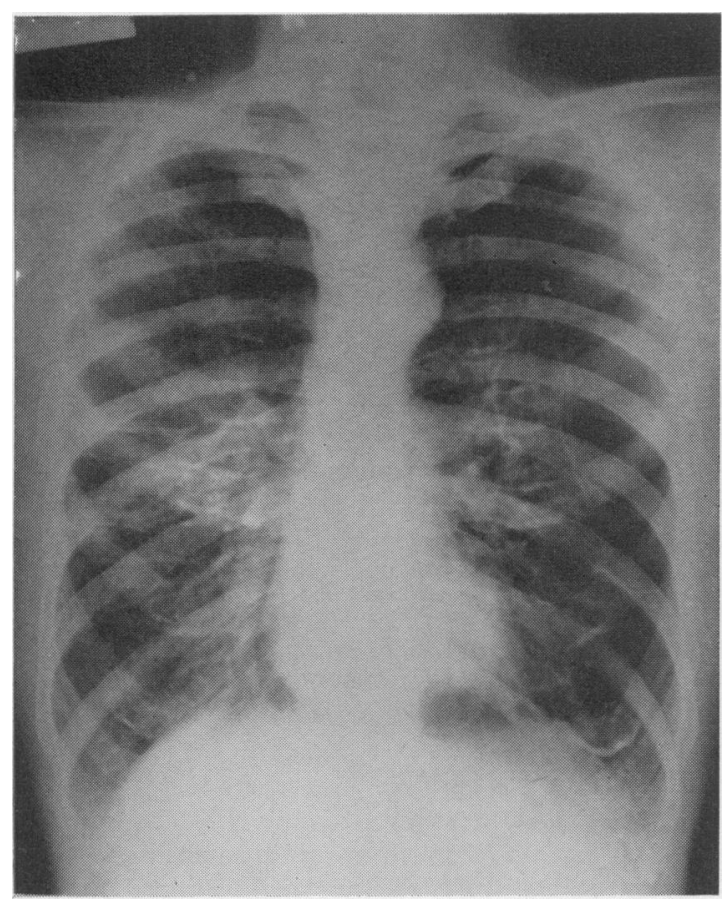

FIG. 3. Bullous emphysema with septa visible between cysts.

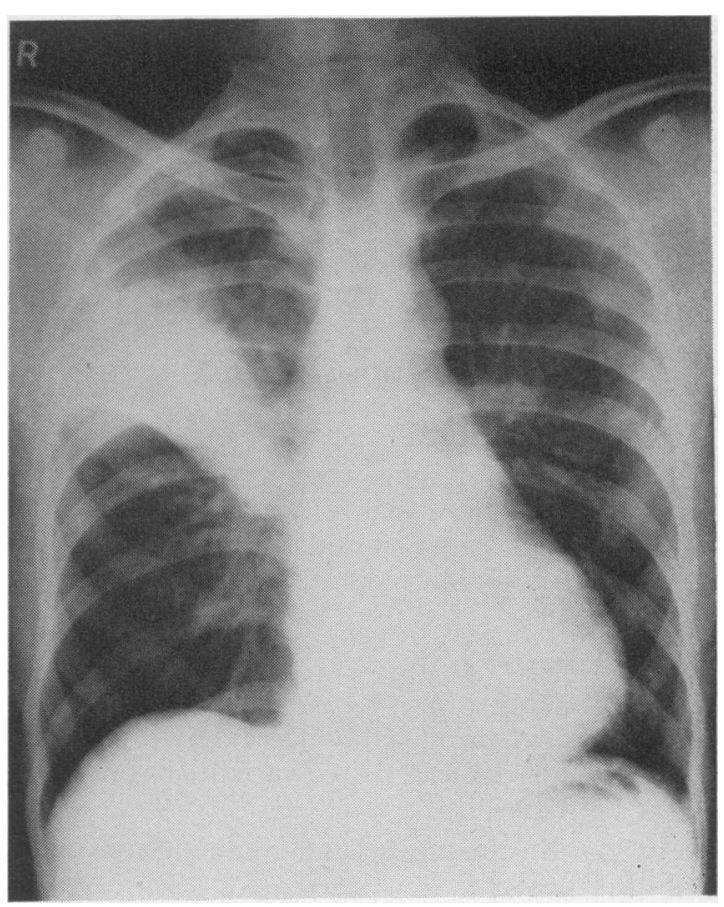

FIG. 4. Consolidation in right upper lobe.

of a pneumothorax, the rupture of a lung abscesse into the pleural cavity, i.e. pyopneumothorax, or the communication of an empyaema with the lung. It may be necessary to differentiate between a hydropneumothorax and a lung abscess. A lateral film will help to localize the site of the fluid level and, on occasions, a film taken in the lateral decubitus position with a horizontal beam will show the fluid just beneath the chest margin running in the pleural cavity.

It is not always possible to recognize underlying lung pathology in either a pneumothorax or a hydropneumothorax. Follow-up films should be closely examined for signs of parenchymal lesions that may be responsible.

\section{Lobar pneumonia}

It is necessary to recognize the classical appearance of lobar consolidation, as it is seen in pneumococcal pneumonia but other causative factors may produce an identical appearance, e.g. fungi, viruses, collagen diseases, reticuloses, neoplasms and even occasionally radiation therapy. Pneumococcal pneumonia may produce no radiological signs for the first $24-48 \mathrm{hr}$, but then exudation into the alveoli results in the development of a dense sharply defined opacity, with a lobar or segmental distribution (Fig. 4). The volume of the affected area may be slightly 
increased and this may cause minimal bulging of adjacent fissures. These may be convex in shape and are distinguishable from the concave fissures which shift towards a collapsed segment. In the presence of consolidation the vascular markings are obliterated and patent air-filled bronchi may be visible coursing through the opaque lung segment. Patent bronchi are not visible in collapsed areas and are not visible when the opacity is due to fluid. The diaphragmatic and mediastinal borders are usually sharply defined and pulmonary consolidation does not result in any shift of the heart or mediastinum. A significant fluid collection may result in shift of the heart to the opposite side and collapse may produce shift to the same side. When consolidation is identified on a postero-anterior chest film, it is necessary to take a lateral film to accurately localize it. An overpenetrated film may be required to identify the translucent bronchi. Lobar pneumonia may be accompanied by a pleural effusion.

\section{Bronchopneumonia (Fig. 5)}

This is characterized by multiple patchy areas of consolidation, usually bilateral and basal but often scattered throughout both lungs or on occasions confined to one side. Rarely areas of lobar consolidation may occur. There are many causative organisms including staphylococci,

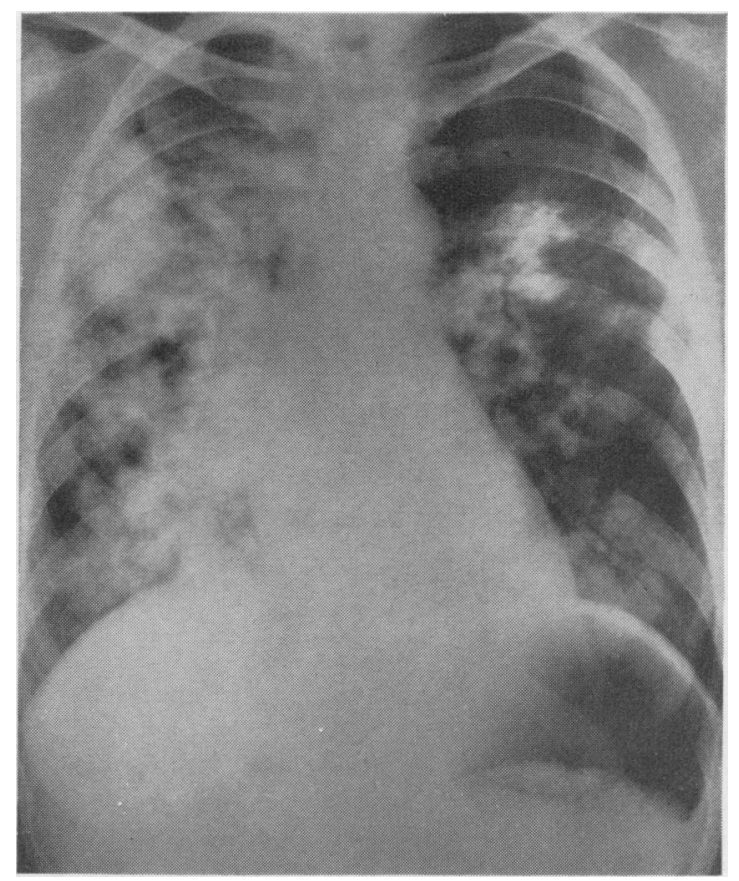

FIG. 5. Patchy bronchopneumonic consolidation in both lungs.

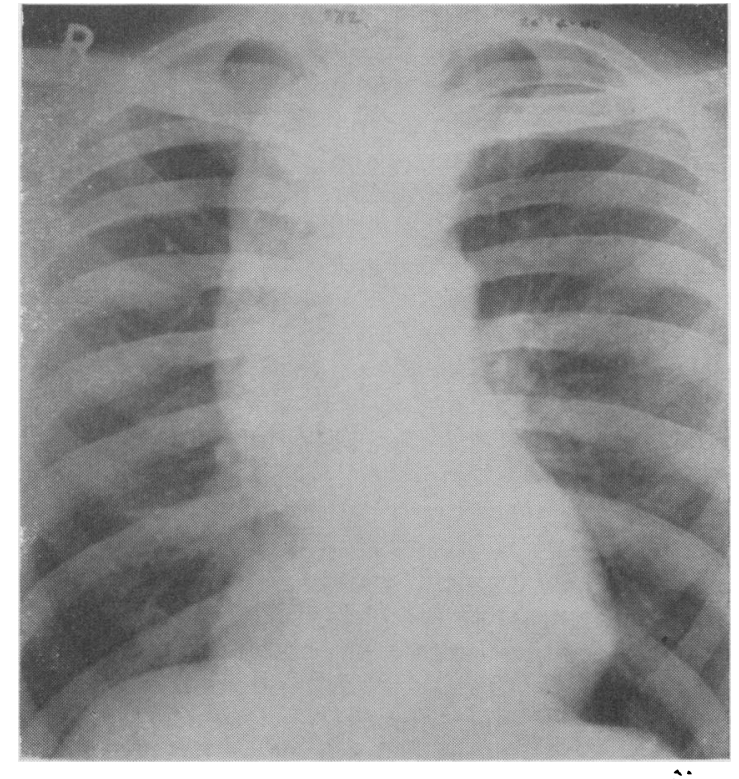

FIG. 6. Widened mediastinal shadow due to achalasia.

Food debris visible in the oesophagus.

streptococci, which may follow epidemics of influenza, whooping cough or measles, tubercle bacillus and Friedlander's bacillus. Pneumatocoeles are especially prone to complicate staphylococcal pneumonia. In children, glandular enlargement in the mediastinum in staphylococcal bronchopneumonia may produce a radiological appearance similar to Hodgkin's disease.

Aspiration pneumonia may occur postoperatively or may complicate oesophageal lesions such as achalasia. Achalasia may be recognized by the convex shadow of the oesophagus bulging to the right of the mediastinum (Fig. 6). Contrast examination of the gullet is indicated in cases of recurrent pulmonary infection to exclude lesions such as pharyngeal or oesophageal diverticula, achalasia, hiatus hernia and oesophageal stricture formation. Chronic aspiration pneumonia can lead to lung fibrosis.

Lobar consolidation or patchy bronchopneumonic consolidation may complicate a proximal bronchial carcinoma.

\section{Lobar collapse}

In the acquired form, bronchial obstruction produces de-aeration of the affected segment.

Bronchial obstruction may be due to: (a) pressure from without, e.g. glandular mass, aneurysm, neoplasm; (b) intrinsic narrowing of the walltuberculous endobronchitis, adenoma, neoplasm ; and (c) obstruction in the lumen-viscid sputum, foreign body, neoplasm. 
Often more than one cause is operative. Compression collapse may result from a large tension pneumothorax or a pleural effusion.

Radiological investigation of suspected collapse should include postero-anterior, lateral and overpenetrated postero-anterior films. Occasionally oblique films or a lordotic view are of value. When the complete lung is collapsed the hemithorax is totally opaque and the trachea and heart are displaced to the affected side. The hemidiaphragm is elevated but it is not possible to recognize its level in the uniform opacity. On the left side it may be noted that air in the stomach is lying at a very high level.

Lobar collapse (Figs. 7 and 8) produces a dense opacity often with shift of the fissures towards the collapsed segment, and also in extreme cases shift of the heart and trachea.

In the upper lobes the postero-anterior film may show an opacity which widens the superior mediastinum. The tracheal deviation to the involved side is an important sign.

Collapse of the middle lobe and lingula produces an ill-defined opacity lateral to the hilum and often merging with the heart shadow, and obscuring its outline. The lateral view is especially helpful as it showed a dense opacity with shift of the fissures. The opacity passes downwards from the hilum towards the sternum.

Collapse of the lower lobe on the right produces a linear opacity passing downwards from the hilum outside the heart border. This is harder to

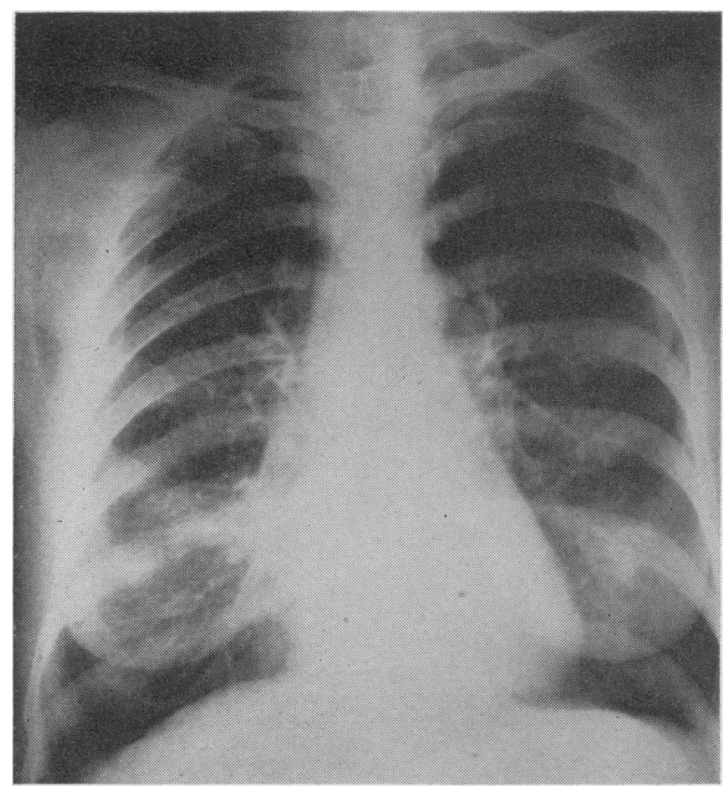

FIG. 7. Ill-defined opacity lateral to the right heart border.

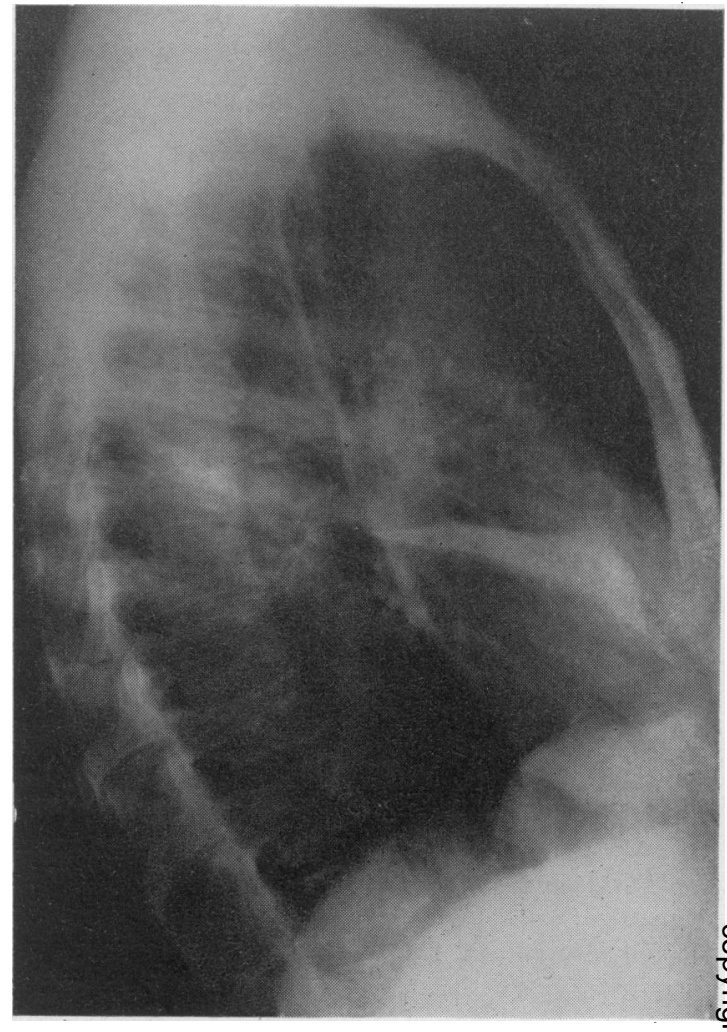

FIG. 8. Lateral film shows opacity to be due to a collapsed middle lobe.

see when involving the left lower lobe as it may lie behind the heart shadow. An overpenetrated film to show this area is most helpful. The collapsed segment may be obvious on the lateral view but occasionally no opacity is seen as this collapsed segment lies in the paravertebral gutter. It is in these cases that a right or left posterior oblique view of the chest is most helpful. Lower lobe collapse is often accompanied by a displacement of the hilum downwards on the affected side.

Often a compensatory expansion of the other lobes occurs in the presence of collapse. This results in an increased translucency of the remaining lung and a 'fanning out' of the vascular markings.

\section{Pleural effusion (Fig. 9)}

Pleural fluid collects primarily in the posterior and lateral parts of the costo-phrenic recess. The earliest radiological sign may be minimal blunting of the costophrenic angles. A small lamellar effusion may pass upwards from the costophrenic angle along the rib margin.

Larger effusions produce a dense opacity which has a concave upper border and is highest later- 


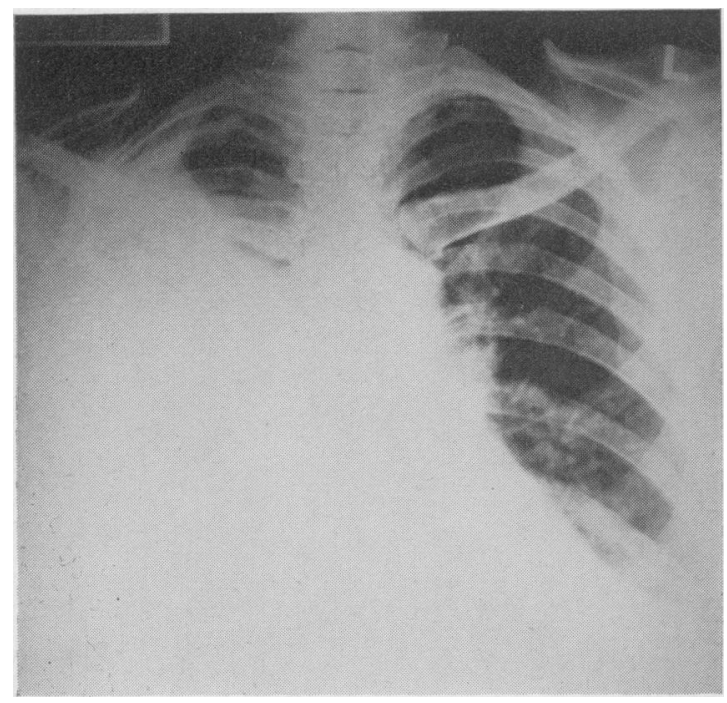

FIG. 9. Large right pleural effusion.

ally. The lateral film shows an opacity which is highest posteriorly. A pleural effusion produces shift of the mediastinum to the opposite side. This may be extreme in the presence of a hemithorax totally opaque due to fluid. The shift of the mediastinum to the opposite side distinguishes fluid from total collapse when the mediastinum passes to the same side. When no shift occurs in the presence of fluid it usually indicates an underlying atelectasis or rarely a rigid mediastinum. Adhesions or collapse may alter the shape of the effusion and even reverse the curve.

In most cases it is not possible to determine the cause of the effusion radiologically, as the underlying lung is obscured. A careful search should, however, be made for any obvious lung, bone or heart disease. There may be evidence of apical tuberculosis, mediastinal glandular enlargement, heart enlargement and vascular engorgement. The ribs may show evidence of lytic or osteoblastic secondary deposits. Pleural effusions frequently complicate lobar or bronchopneumonia. There are many causes of pleural effusion which may give no other radiological evidence in the chest, e.g. Meig's syndrome, collagen diseases and nephrotic syndrome. Fluid may spread into the fissures producing spindle-shaped deformities visible in the oblique or horizontal fissures on the lateral film (Fig. 10). These may produce ill-defined opacities in the line of the fissures in the postero-anterior view.

Interlobar effusions may be found in the absence of a free pleural effusion.

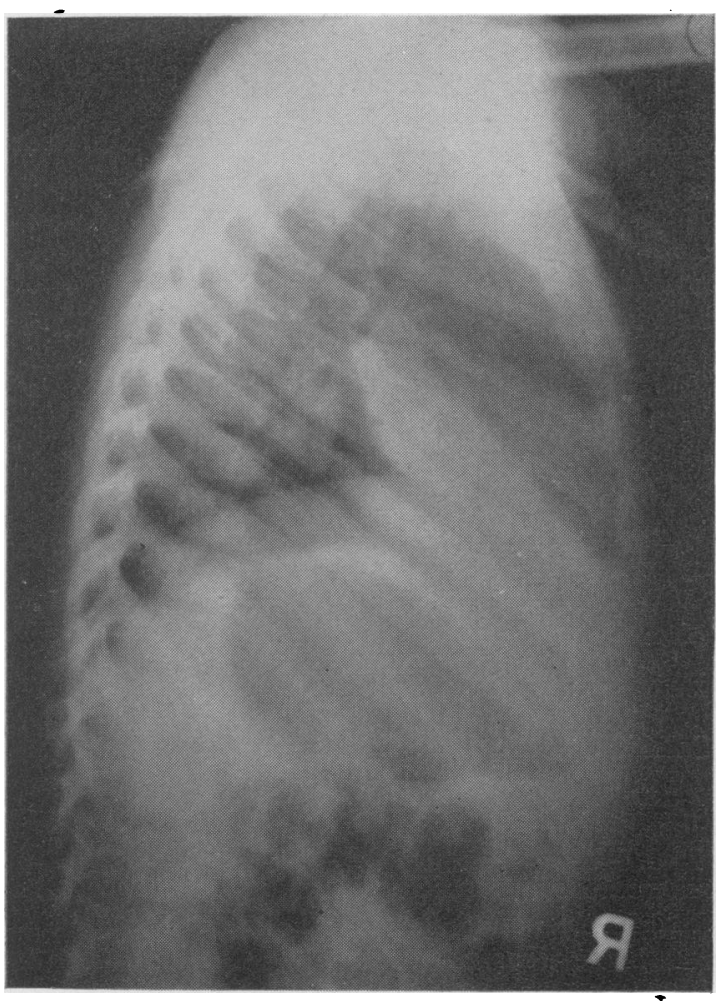

FIG. 10. Spindle-shaped interlobar effusion in the oblique fissure.

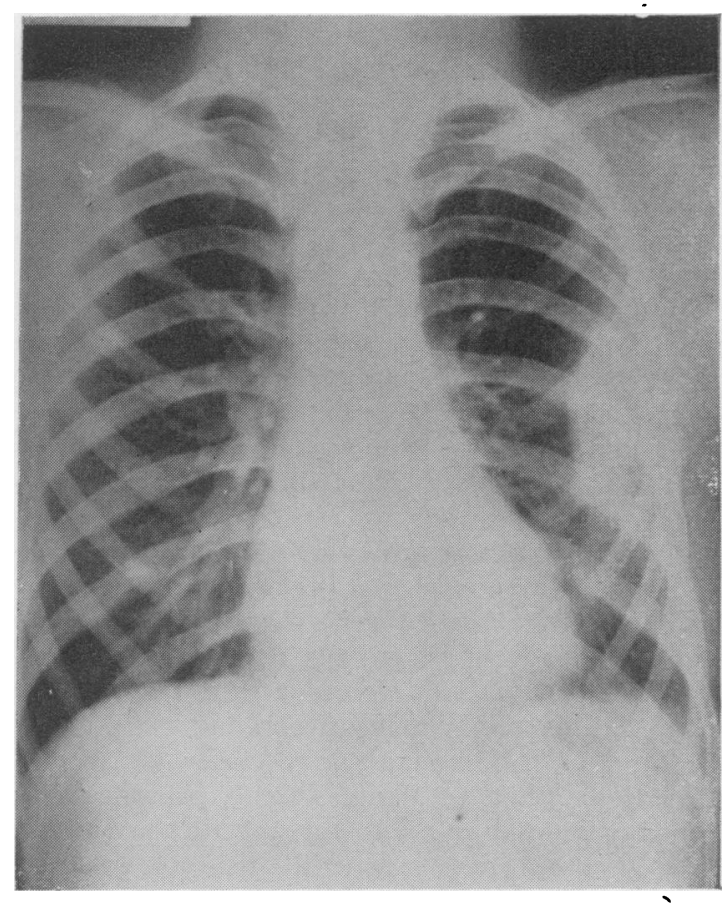

Fig. 11. Encysted lateral parietal effusion. 


\section{Encysted effusion (Fig. 11)}

Encysted effusions may be: (a) parietal, (b) interlobar, and (c) mediastinal. The most common variety is a parietal effusion and usually situated: (i) laterally along the chest wall, or (ii) posteriorly overlying the spine. The opacity is dense and in the lateral view well demarcated and convex toward the lung. In the postero-anterior view the density of the opacity may be slightly less in its upper part.

Mediastinal effusions are not uncommon following cardiac surgery. Rarely they become encysted.

Tapping of an encysted effusion may inadvertently introduce air and the radiological appearances may simulate a lung abscess.

Posteriorly encysted pleural effusions may have to be differentiated from carcinoma, neurofibroma and neurenteric cysts.

\section{Calcified pleura (Fig. 12)}

This usually produces a characteristic appearance of bizarre dense areas of calcification overlying the lung field. The most common causes are tuberculous pleural effusions and haemothorax. Calcification of the pleura can occur in fine linear streaks in asbestosis.

\section{Tuberculosis (Fig. 13)}

The radiological appearances of pulmonary tuberculosis are so varied as to be outside the scope of this paper. Suffice it to say that the following may occur: lobar consolidation, bronchopneumonia, infiltration, patchy irregular upper zone infiltration with cavitation or linear fibrosis. Single round opacities due to tuberculomata may simulate primary peripheral carcinoma or a secondary deposit.

Mediastinal glandular enlargement in this country was invariably seen only in children in primary infection. In immigrants adult glandular mediastinal tuberculosis is not infrequently seen. It is important for the Casualty Officer to be familiar with miliary tuberculosis and its main differential diagnosis.

In miliary tuberculosis the lungs are covered with multiple small opacities of approximately $1 \mathrm{~mm}$ in size. Usually these are maximal in the upper zones and fade off towards the base. Usually there is no pleural effusion. Although miliary tuberculosis may affect adults, it is most common in children and may rarely be accompanied by enlarged mediastinal glands. With bronchopneumonic spread the opacities are larger, i.e. $2-4 \mathrm{~mm}$. These frequently tend to coalesce. Cavitation may occur early in the bronchopneumonic form. Miliary tuberculosis may have

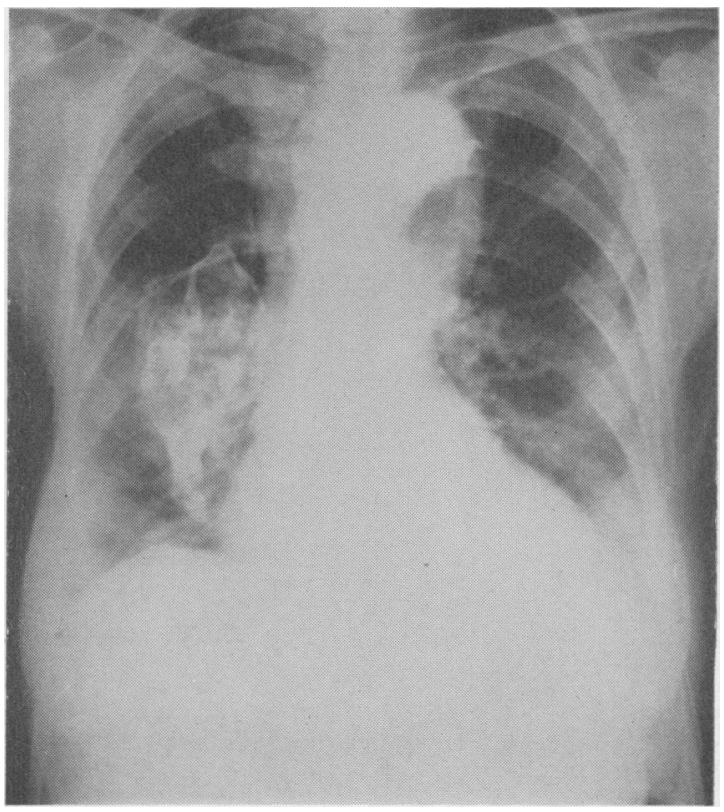

FIG. 12. Bizarre calcification - pleural in origin.

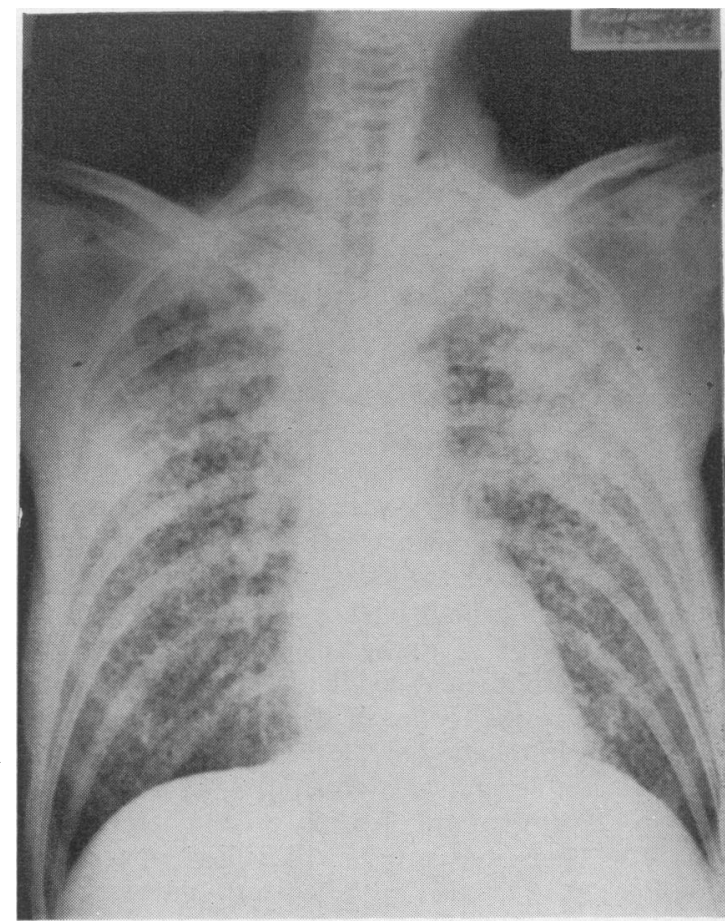

Fig. 13. Tuberculosis. Multiple opacities are superimposed over the upper zones. 


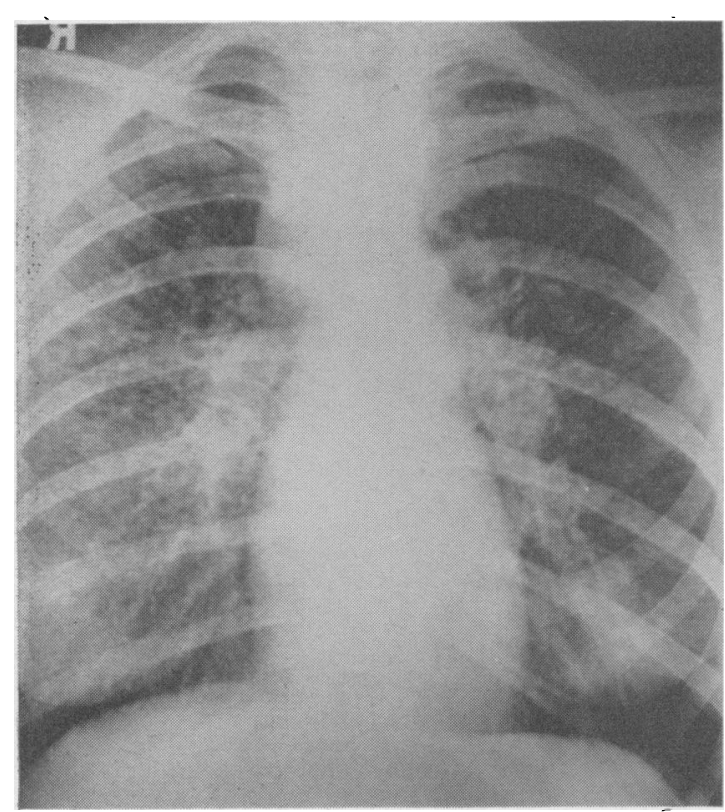

FIG. 14. Sarcoidosis. Linear and nodular lung opacities. Peribronchial glandular enlargement and one right paratracheal gland enlarged.

to be distinguished from other causes of bilateral lung shadowing such as:

\section{(a) Sarcoidosis (Fig. 14)}

In this the glandular enlargement is frequently the earliest manifestation. The glands have a peribronchial distribution and are most often enlarged bilaterally. Although the peribronchial enlargement predominates, paratracheal glandular enlargement may also occur.

Pulmonary infiltrations vary, and may consist of a coarse linear infiltration with or without nodular opacities. The nodules may vary in size from millet seed opacities $0.5 \mathrm{~mm}$ in diameter to larger opacities $2-\mathbf{3} \mathrm{mm}$ in diameter. Diagnosis is easy when only peribronchial glandular enlargement occurs but is relatively difficult when pulmonary infiltrations occur without any glandular enlargement.

\section{(b) Pneumoconiosis (Fig. 15)}

Three stages can be recognized in pneumoconiosis: (i) pre-nodular, (ii) nodular, and (iii) progressive massive fibrosis.

The appearances in the lung fields vary greatly depending on the dust responsible, and varying degrees of nodulation or reticulation may occur. The linear reticulation may consist of lines A which are coarse linear opacities radiating from the hilum, lines B which are short narrow lines found at the bases and passing transversely to end at the pleural margin, and lines $\mathrm{C}$ which are fine lines producing a network shadowing over the lungs. They are, respectively, due to involvement of the deep, intercommunicating and superficial lymphatics. Diffuse linear shadowing is not a characteristic of tuberculosis. The nodules vary greatly in size and usually commence around the hilum. In the stage of progressive massive fibrosis large opacities occur in the upper zones accompanied by a great deal of fibrosis. The appearances may be due to associated tuberculosis in some cases, but this is not invariable. In difficult cases, the history of an industrial dust exposure is most helpful. Where the radiological appearances would include this, the patient should be questioned for both his present and past occupations.

\section{(c) Secondary deposits (Fig. 16)}

These opacities characteristically vary in size and are maximal at the bases. They are larger than the millet seed opacities of miliary tuberculosis.

\section{(d) Haemosiderosis (Fig. 17)}

The most common form is the secondary type. This is usually a complication of mitral valve disease or less often other causes of recurrent left heart failure. The opacities are usually maximal

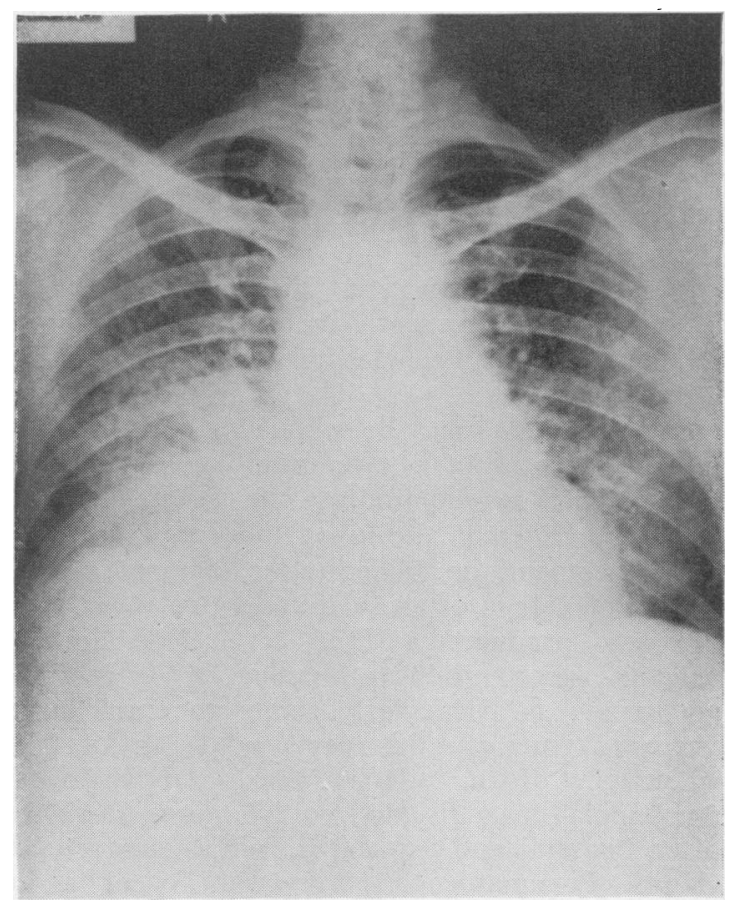

Fig. 15. Pneumoconioisis. Peri-hilar and basal distribution. 


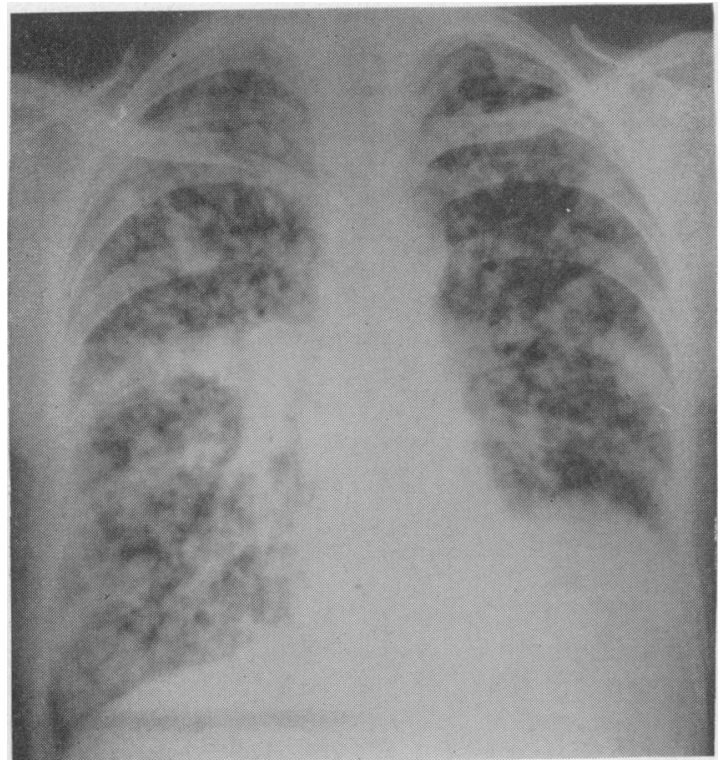

Fig. 16. Carcinomatosis. Large basal opacities present.

around the hilum and at the bases. The characteristic contour of mitral valve disease is readily recognized.

\section{(e) Lymphangitis carcinomatosa and Hamman- Rich Syndrome}

These usually produce streaky shadowing with little if any nodulation.

Lines $\mathbf{A}$ and $\mathbf{B}$ seen in pneumoconiosis are also often seen in lymphangitis carcinomatosa.

\section{Lung abscess (Fig. 18)}

Lung abscess may result from: (a) aspiration, (b) pneumonia, (c) septic emboli, (d) carcinoma, (e) infection in bronchiectasis, and (f) fungus infections.

Lung abscesses may be solitary or multiple. The more common solitary type usually commences as an area of consolidation on the right side. It is seen most often in the lower lobes especially the apical segment, or the posterior segment of the upper lobe. It is often surrounded by an area of Necrosis produces a fluid level. Friedlander's oedema which produces blurring of its margin. pneumonia is particularly liable to form large abscess cavities and this may produce an increase in volume of the affected area. Staphylococcal pneumonia gives rise to multiple lung abscesses often bilateral and associated with enlarged hilar glands. Pneumatocoeles commonly occur as a result of staphylococcal infection. Other complications of abscess formation are tension cavities,

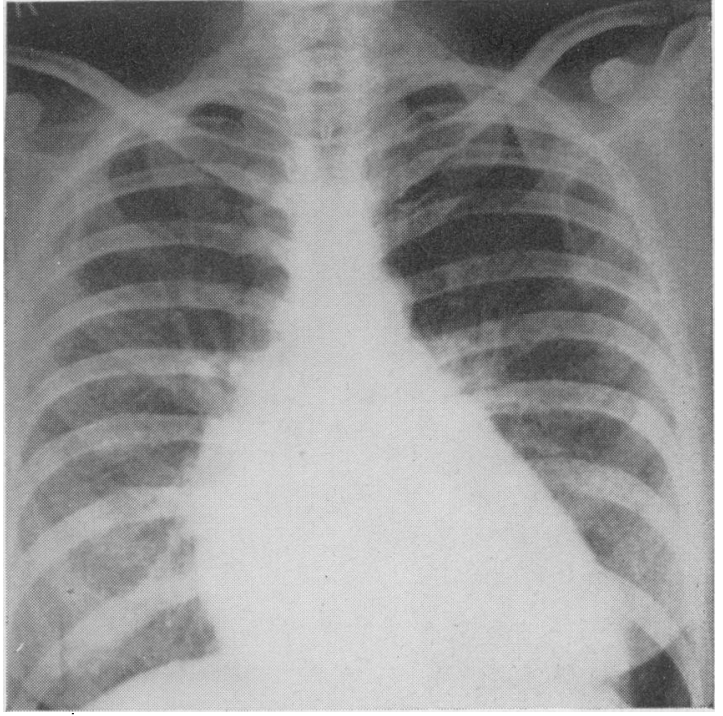

FIg. 17. Haemosiderosis. Fine peri-hilar and basal shadowing.

pleural effusion, interlobar effusion, empyema and pyopneumothorax.

Radiological investigation includes a postero $\frac{0}{0} \overrightarrow{\mathscr{O}}$ anterior film and a lateral for accurate localiza-. tion. An overpenetrated postero-anterior film is often helpful to study the cavity wall and the hilar regions for signs of glandular or neoplastic masses. Tomography may be necessary to study the cavity wall. In cases of cavitating neoplasm

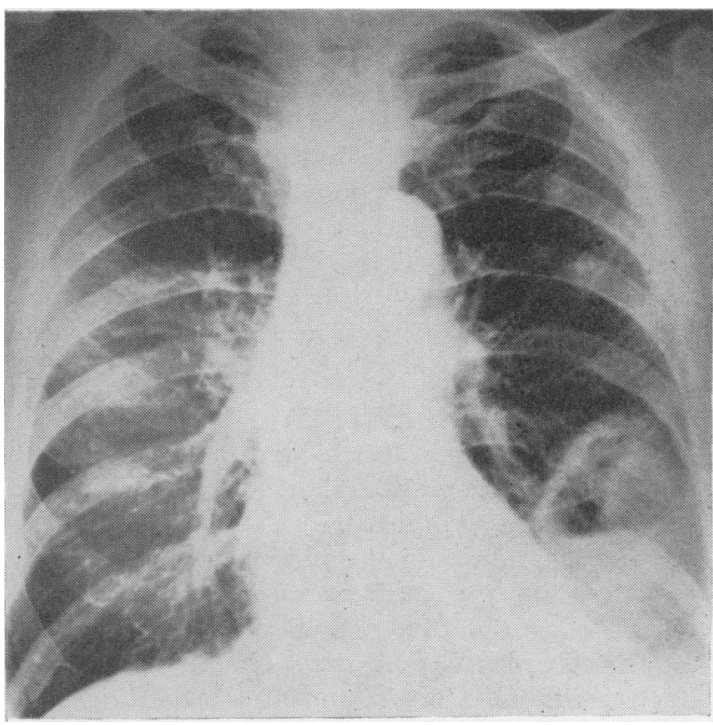

FIG. 18. Lung abscess left lower lobe with fluid level. 
the wall is thickened and irregular and has multiple polypoid-like areas projecting into the cavity. An area of adjacent atelectasis or streaky shadowing may be indicative of malignancy. Fungus infection in a pre-existing cavity will produce a mycetoma which often has a crescent-shaped air translucency capping the opacity lying in the cavity.

\section{Carcinoma (Figs. 19 and 20)}

There are many ways in which bronchial carcinoma may present radiologically. The following may be seen:

(1) An irregular mass in the hilum.

(2) A peripheral round or irregular opacity.

(3) An area of atelectasis with or without an associated hilar mass.

(4) An abscess distal to a hilar mass which may or may not be obvious radiologically.

(5) An area of pneumonic consolidation distal to a proximal neoplasm.

(6) A cavitating peripheral mass.

(7) Obstructive emphysema.

Other complications of bronchial carcinoma may dominate the picture, such as pleural effusion, pericardial effusion, invasion of a rib directly or by deposit.

A peripheral bronchial carcinoma often presents late and may even be found incidentally. It may be round or oval and well demarcated. This type of lesion has to be differentiated from other causes of a 'coin lesion' such as tuberculoma, secondary deposit, hamartoma, arteriovenous malformation, adenoma or lung abscess. Tomography can greatly assist in the differentiation. The opacity may have irregular streaky linear opacities around it due to lymphangitis carcinomatosa or direct neoplastic infiltration. The presence of a notch or 'hilum' in the opacity is said to strongly favour a primary malignant lesion. Calcification in the opacity is strongly against a primary bronchial neoplasm and suggestive of a tuberculoma or a hamartoma. 'Pop-corn' calcification suggests hamartoma. Satellite nodules may be seen in tuberculoma. The 'feeding' vessel may be seen in arterio-venous malformation. Tomography of the hilum may be necessary to distinguish between a neoplastic mass, a glandular mass or a vascular opacity. The finding of an area of bronchial stenosis may indicate neoplastic infiltration.

If elevation of one dome of the diaphragm is present then screening will be helpful to detect paradoxical movement common with diaphragmatic paralysis.

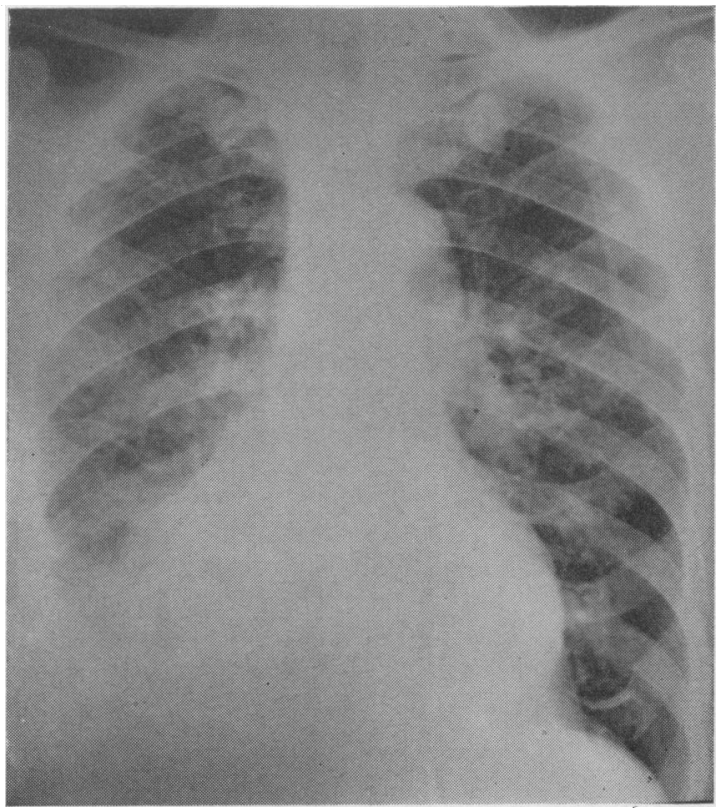

FIG. 19. Collapse consolidation of right lower lobe with pleural effusion due to carcinoma.

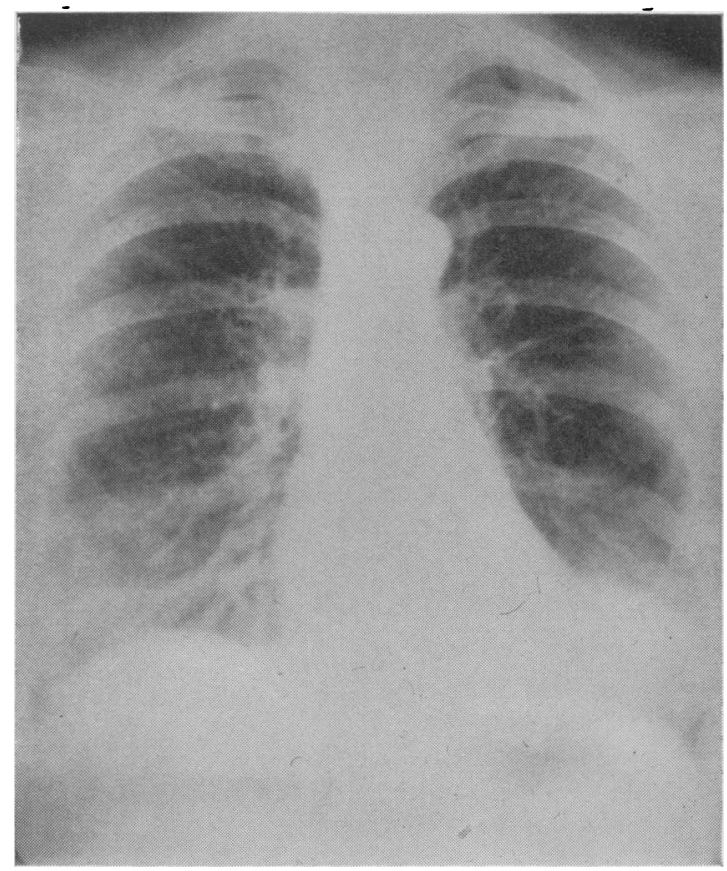

Fig. 20. Peripheral carcinoma left lower lobe. 


\section{Mediastinal masses}

Occasionally a patient may present in the casualty department with chest pain, dyspnoea, dysphagia, cyanosis, venous engorgement in the neck and oedema of the upper limbs and neck. These changes can be produced by large masses in the superior mediastinum. The most common cause for this is glandular enlargement due to Hodgkin's disease, lymphosarcoma, reticulum cell sarcoma or rarely lymphatic leukaemia. Rarely a bronchial carcinoma may produce massive glandular enlargement with compression. Other causes of superior mediastinal masses such as retrosternal thyroid, dermoids, teratoma, thymoma, neurogenic cysts and aortic aneurysms may rarely be large enough to produce these symptoms.

Radiological investigation consists of a posteroanterior film and a lateral view. Glandular enlargement is most often bilateral and presents a characteristic lobulated outline (Fig. 21). The single solid masses may be differentiated by their position in the mediastinum. In the anterior mediastinum, thyroid, dermoid, teratoma and thymoma are most common. Calcification may be seen in thyroid adenoma and thymoma. There may be bone or dental elements in dermoids. Glandular masses and aneurysms arise in the middle mediastinum but may grow into the anterior compartment. A posteriorly situated mass is usually a neurogenic tumour or more rarely a neurenteric cyst.

Further examinations such as barium studies, screening for pulsation and angiography may be necessary. Tomography may clearly show the bilateral lobulated character of glandular masses.

The reticuloses may produce secondary changes

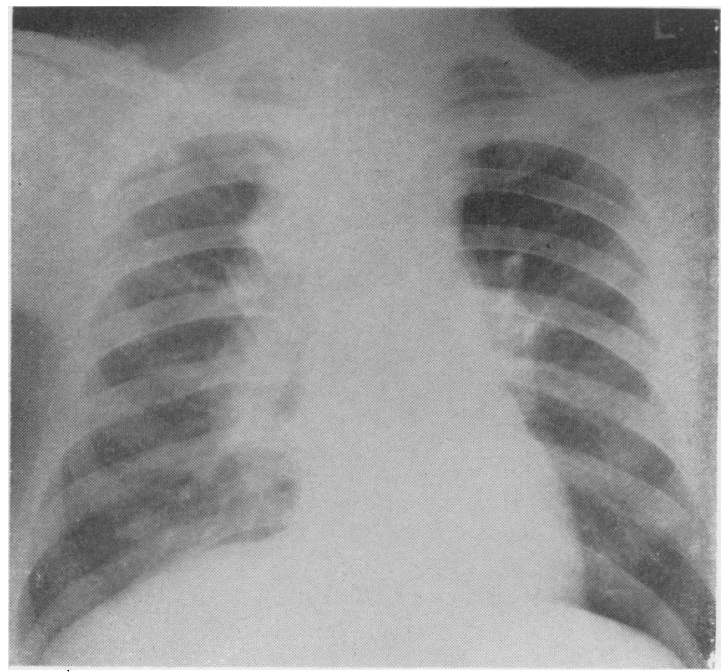

Fıg. 21. Hodgkin's glands in superior mediastinum.

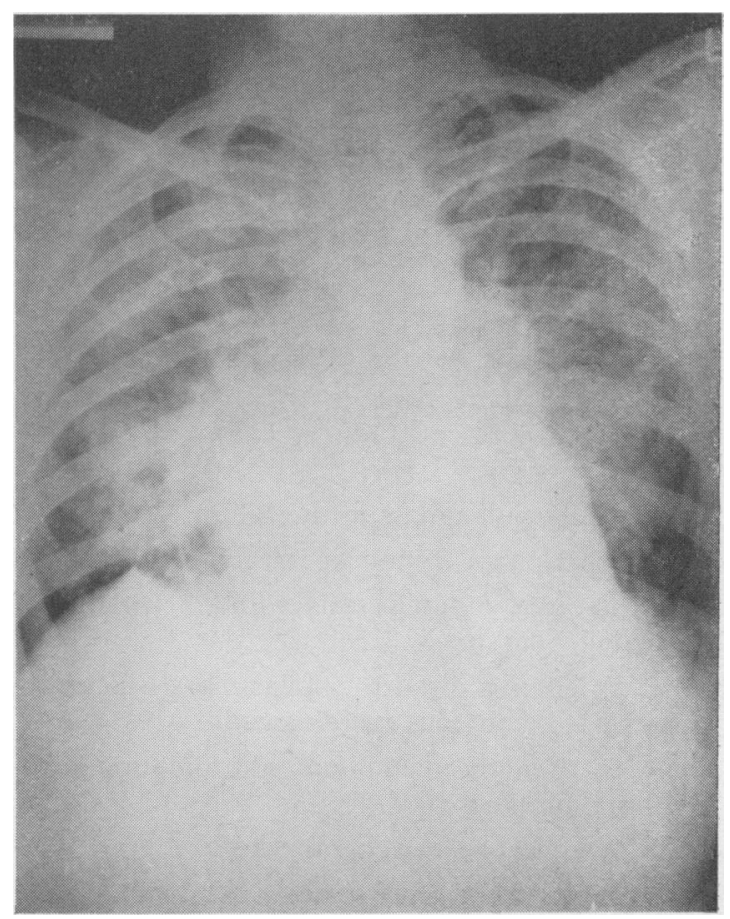

FIG. 22. Pulmonary oedema. Butterfly perihilar shadowing, sparing apices, bases and periphery.

in the lungs, such as streaky linear infiltration, patchy nodular opacities, which may even cavitate, or areas of atelectasis or consolidation.

Tuberculosis rarely causes massive paratracheal glandular enlargement in natives of this country, but is being seen more often nowadays in immigrants.

\section{Left heart failure}

In acute cases, pulmonary oedema may occur. This presents as a bilateral peri-hilar shadowing referred to as butterfly or bat's-wing shadowing (Fig. 22). It spares the apices, bases and the periphery. Rarely, unilateral pulmonary oedema may be seen.

Chronic heart failure or the early stages of left heart failure may produce vascular engorgement in the hila and lungs, septal B lines at the bases, and pleural effusion. The outflow tract of the right ventricle may be prominent. A common cause for this is mitral stenosis (Fig. 23). In this the pulmonary outflow tract is prominent, there is enlargement of the left atrium which may produce a small bulge on the left heart border below the pulmonary artery, and a double shadow behind the right heart border. The left atrium can be so large as to form part of the right heart border 


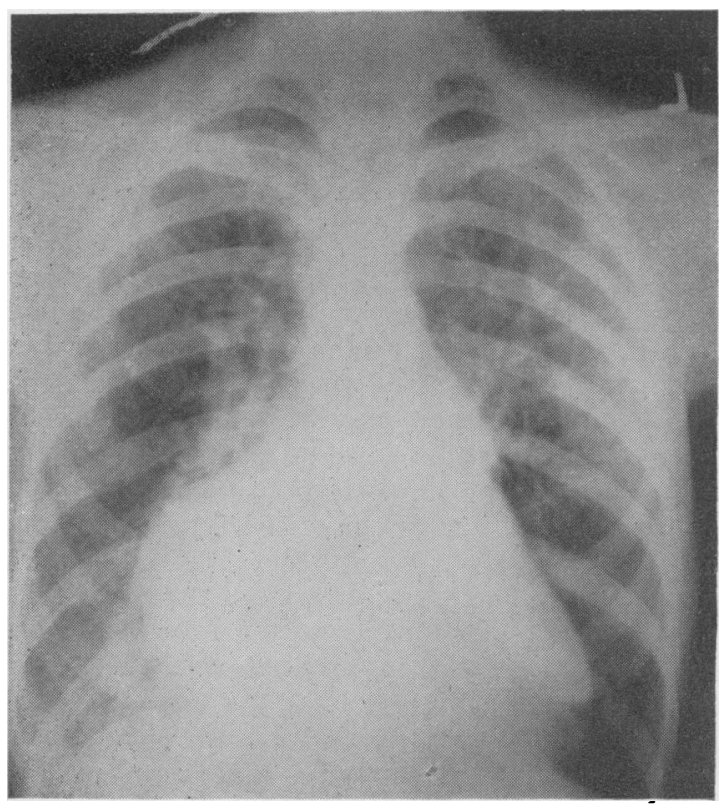

FIG. 23. Mitral heart disease with aneurysmal enlargement of the left atrium, enlargement of upper lobe vessels, prominence of pulmonary conus segment, and septal B lines.

radiologically, or even to extend beyond it. An overpenetrated postero-anterior film may show widening of the carina. Calcification may occasionally be seen in the valve.

When right heart failure occurs the superior vena cava may enlarge and widen the mediastinum. There may also be right atrial enlargement and engorgement of the liver.

To determine specific chamber enlargement it may be necessary to take oblique views. Screening and barium swallows are helpful to show the nature and the extent of the enlargement.

\section{Dissecting aneurysm (Fig. 24)}

Although this may be chronic, it is more common for dissecting aneurysm to present acutely with severe chest pain and shock. The clinical picture may strongly mimic coronary thrombosis. The dissection may spread proximally into the pericardial sac and produce death from cardiac tamponade. It may spread distally down the thoracic aorta and produce occlusion of the major aortic arch vessels.

Postero-anterior and lateral films should be obtained if possible, but frequently the first $\mathrm{X}$-ray is an antero-posterior portable film on the ward. This projection unfortunately often exaggerates the width of the mediastinum.
In cases of dissection the aorta is dilated and widened. It often has an ill-defined border and an accompanying mediastinal reaction produces a hazy mediastinal outline. There is frequently a left pleural effusion, which may be blood-stained. The aortic knuckle may be elevated and displaced to the left. Loss of parallelism of the aortic walls and local 'bulges' are very suggestive signs. The radiological signs, however, are often very slight and contrast examinations may be necessary for confirmation. It is helpful if previous chest X-rays are available for comparison. Dissecting aneurysms may lead to enlargement of the left ventricle.

A dissection can become chronic and there may even be calcification visible in the false passage. The mortality rate is exceedingly high, but the results of operative treatment are improving.

\section{Pericardial effusion (Fig. 25)}

There are many causes of pericardial effusion amongst which the following need consideration: (1) rheumatic disease, (2) acute viral infection, (3) collagen disorders, D.L.E., etc., (4) coronary infarction, (5) tuberculosis, (6) acute septicaemic conditions, (7) nephritis, nephrosis and uraemia, (8) secondary to neoplastic disease, (9) myxoedema, and (10) trauma.

The fluid lies in the postero-inferior recess and may pass undetected radiologically. At this stage the opacity caused by the inferior vena cava, best

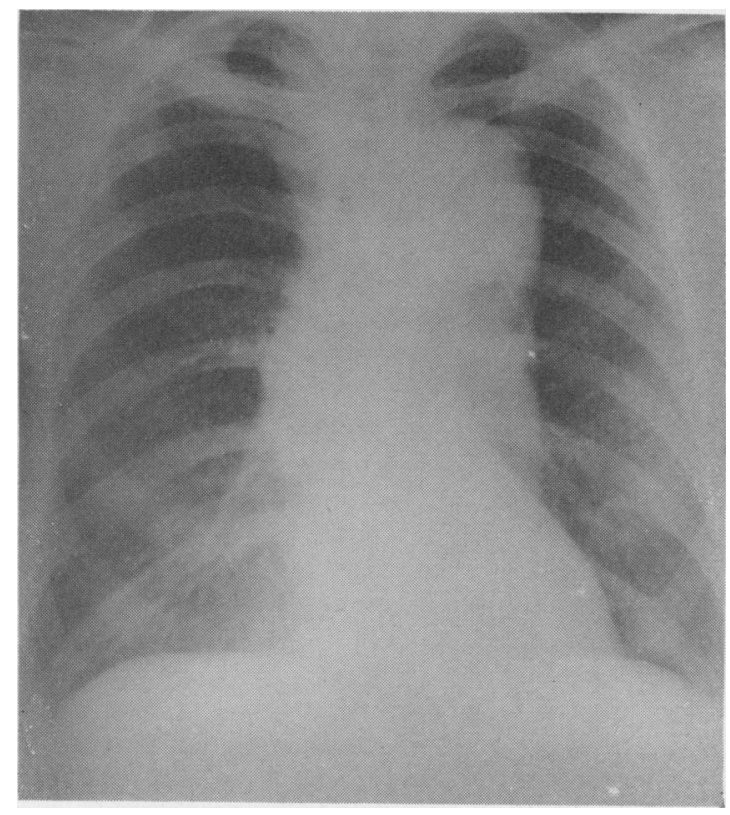

FIG. 24. Dissecting aneurysm of the aorta. 


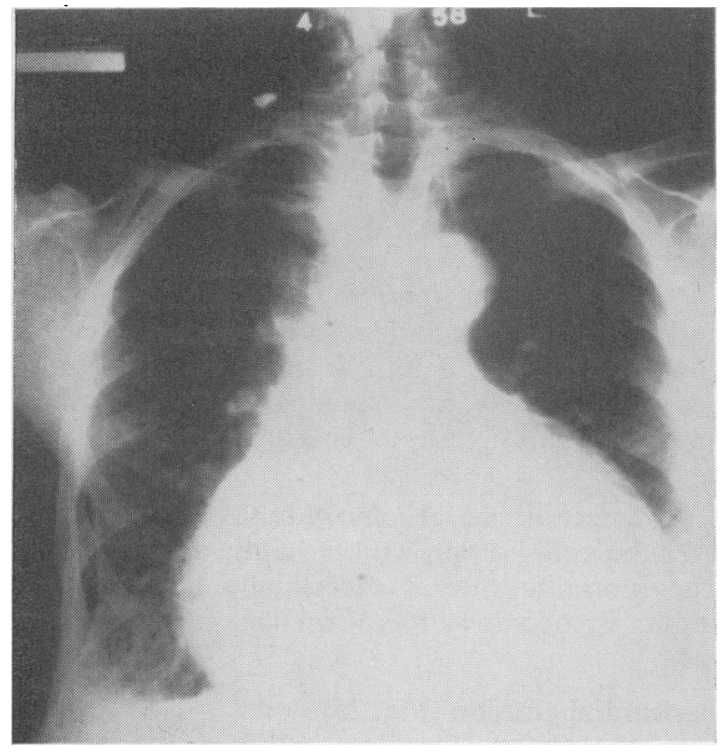

FIG. 25. Overpenetrated film to show cardiac shape in pericardial effusion.

seen on the lateral view, is obliterated. As more fluid accumulates the normal contours of the heart are altered, the borders are straightened and then the heart becomes globular in shape. Both cardiophrenic angles remain acute. A barium swallow will demonstrate displacement of the oesophagus backwards. A film taken supine may occasionally show bulging of the cardiac waist by mobile fluid as compared to the appearance on an erect postero-anterior film. Although screening is frequently performed in these cases to demonstrate a diminished amplitude of cardiac pulsation, this may also occur with myocarditis which is one of the main conditions in the differential diagnosis. In difficult cases it may be necessary to perform special procedures to diagnose pericardial effusion. These consist of cardiac catheterization, which alone may demonstrate the 'thickness of tissue' beyond the arterial wall, angiocardiography and sometimes carbon dioxide injection into the heart chambers.

Eventually the effusion may lead to right heart failure with dilatation of the superior vena cava, engorgement of neck veins and enlargement of the liver. Pulmonary congestion may also be present.

\section{Cerebro-vascular accidents}

The diagnosis of cerebral embolus, thrombosis or haemorrhage when suspected clinically is usually confirmed or refuted by angiography. It is often necessary to primarily exclude an intra- cranial tumour, and for this the first investigation is usually a skull $X$-ray. In all these cases it is wise to also obtain a chest $\mathrm{X}$-ray to exclude a primary bronchogenic carcinoma which may produce an intracerebral metastasis. It is difficult for the non-radiologist to interpret skull $\mathrm{X}$-rays but the following points may be noted.

The choroid plexus is frequently calcified on both sides and shows as symmetrical mottled opacities above and behind the pituitary fossa. Rarely the choroid may calcify on one side only. Irregular calcification may be seen in a tumour mass and this applies to both glioma and meningioma. Meningioma may produce changes in the overlying bone. The common sites for meningioma are along the sagittal suture line, the sphenoidal ridges, the olfactory grooves, in the parasellar region and in relationship to the falx. The most common change is an area of thickening and sclerosis in the bone overlying the tumour. There may even be superficial spicule formation. The grooves for the meningeal vessels seen on the plain skull films may be enlarged. Rarely menin-

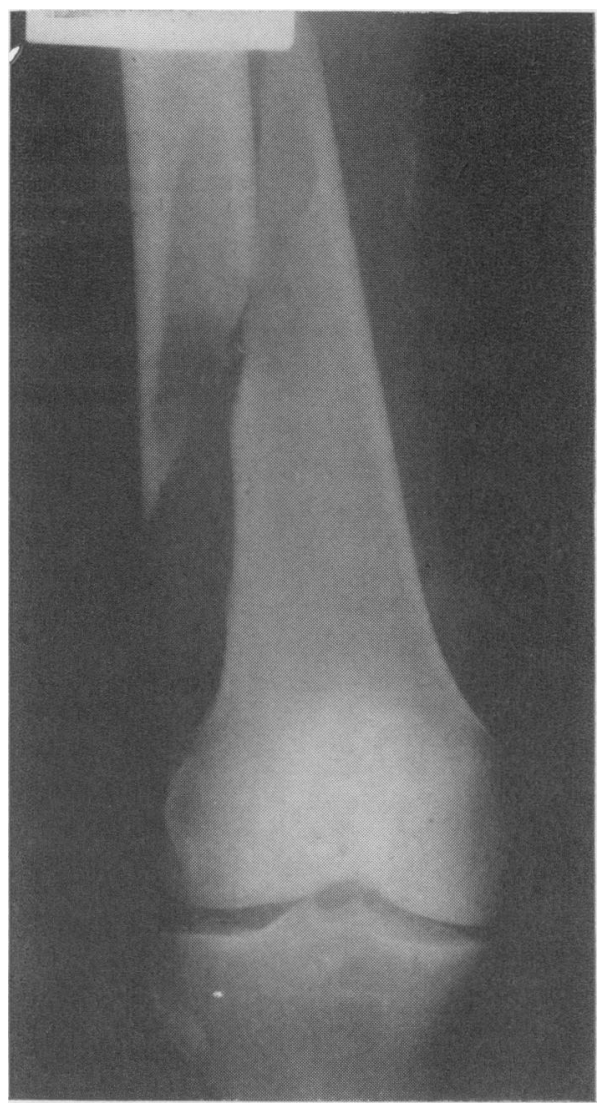

Fig. 26. Fractured femur. Edges show patchy rarefaction due to osteolytic metastasis. 
gioma may produce an area of skull rarefaction overlying the tumour.

Other signs of intracranial masses may be seen, such as a shift of the calcified pineal gland more than $3 \mathrm{~mm}$ to one side. Raised intracranial pressure may produce changes in the sella turcica. There may be erosion of the tips of the posterior clinoids. The dorsum sellae is not usually thinned in these cases. In cases of intrasellar masses the dorsum sellae may be thinned but the posterior clinoids are preserved. Intrasellar lesions also deepen the floor of the pituitary fossa often asymmetrically and undermine the anterior clinoid processes. When raised intracranial pressure deepens the floor of the fossa, it is usually a symmetrical change. Routine skull views required in cases of suspected intracranial lesions include:

(1) Postero-anterior skull view.

(2) Lateral projection.

(3) Towne's view. This is an antero-posterior view with the chin well flexed. It is the best view on which to judge pineal shift and also to see the presence of bilateral choroid calcification.

(4) Submento-vertical view. This demonstrates the base of the skull.

Special areas to examine are: the foramen spinosum which may enlarge in the presence of a meningioma or an anterio-venus malformation,

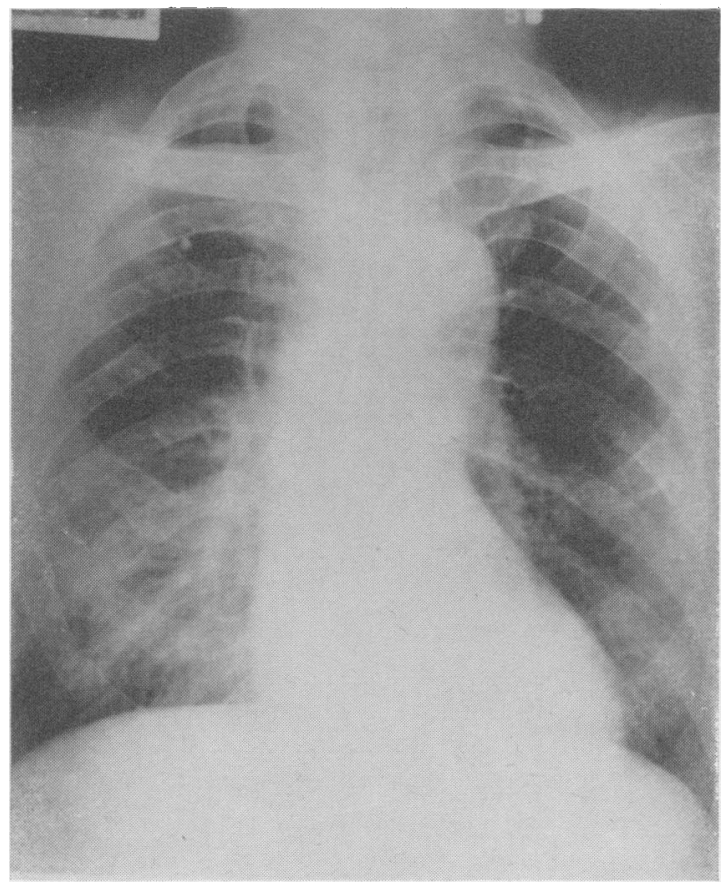

FIG. 27. Chest X-ray showing lytic expanding rib lesions due to multiple myeloma.

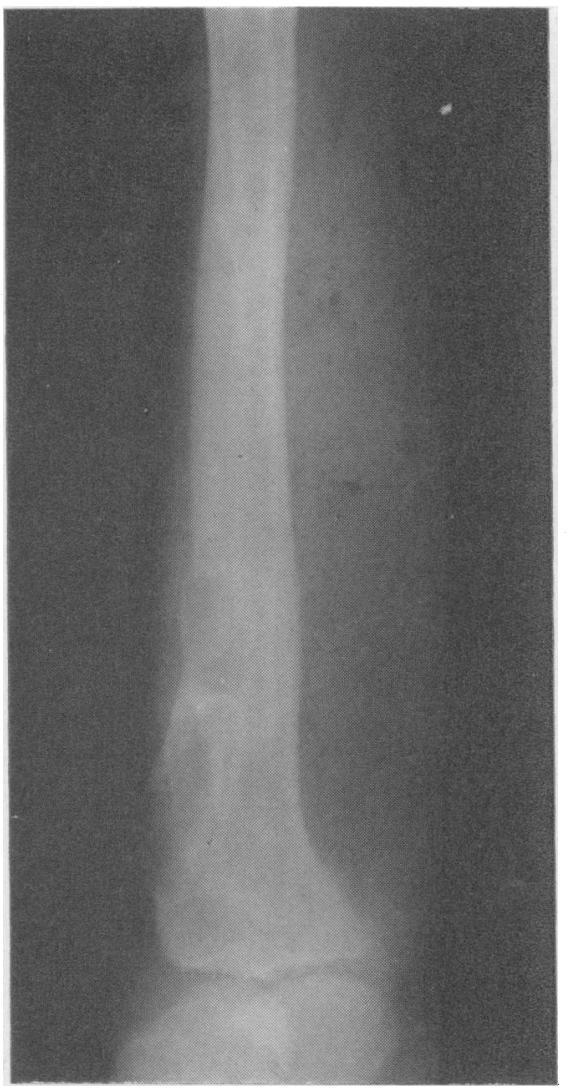

Fig. 28. Cyst-like areas in the tibia due to hyperparathyroidism.

the nasopharyngeal region which can be closely examined in cases where nasopharyngeal neoplasms are suspected and the area of the jugular foramen which may show erosions if a glomus jugulare tumour is present.

If the pituitary fossa appears unusual then special coned views can be taken. It should be remembered that some thinning of the dorsum sellae may be within normal limits in patients over 60 years of age.

\section{Bone disease}

It would be impossible in the space available to discuss the many features of 'bone disease'. However, three conditions will be mentioned which may present as a pathological fracture, namely, secondary deposits, myelomatosis and hyperparathyroidism. The edges of any fracture should be carefully examined for signs of irregular rarefaction, and the surrounding bone for signs of lytic or osteoblastic lesions. If any doubt exists then a skeletal survey should be performed. This 
will consist of X-rays of the skull, proximal long bones, spine, ribs and pelvis. Secondary deposits may be osteolytic, osteoblastic or mixed (Fig. 26). Irregular areas of rarefaction may be seen involving ribs, pelvis, femora, humeri or spine. There may be areas of vertebral collapse with preservation of the disc spaces. The deposits often involve the pedicles of the vertebrae. Except in very advanced cases, the peripheral bones are spared. Myelomatosis involves the medullary bone and may produce scalloping of the cortex from within. There may be multiple lytic areas in the ribs and these may produce expansion (Fig. 27). Soft tissue shadowing may occur with the rib lesions and also with the vertebral lesions and this appearance is almost pathognomonic. When myeloma involves the vertebrae the pedicles are usually spared. Rounded lytic skull lesions are often seen, and the mandible is involved more often by myeloma than by secondary carcinoma.

Hyperparathyroidism (Fig. 28) may produce multiple cyst-like lesions, often resembling giant cell tumours when single, or fibrous dysplasia or even deposits when multiple. There may be an overall bony rarefaction. This also can be the sole manifestation of carcinomatosis or myelomatosis. A diagnostic feature of hyperparathyroidism is the presence of subperiosteal resorption seen most often in the middle phalanges of the fingers. In extreme cases it can even involve the long bones. The skull may show a 'speckled osteoporosis' and there may be loss of the lamina dura of the teeth.

\section{Summary}

The radiological features of some common medical conditions involving the lungs, heart and bones which may present in the casualty department are described. A brief reference is made to the necessary films required as a first step in the radiological investigation.

\section{Acknowledgments}

I wish to thank Dr P. Cardew and the staff of the Photographic Department, St Mary's Hospital Medical School, for their great help in preparing the prints of the X-rays. 